\title{
Comparison of a reduced carbohydrate and reduced fat diet for $L D L, H D L$, and VLDL subclasses during 9-months of weight maintenance subsequent to weight loss
}

\author{
James D LeCheminant*1, Bryan K Smith², Eric C Westman ${ }^{3}$, Mary C Vernon ${ }^{4}$ and Joseph E Donnelly 5
}

\begin{abstract}
Objectives: This study compared LDL, HDL, and VLDL subclasses in overweight or obese adults consuming either a reduced carbohydrate (RC) or reduced fat (RF) weight maintenance diet for 9 months following significant weight loss.

Methods: Thirty-five (21 RC; $14 \mathrm{RF)} \mathrm{overweight} \mathrm{or} \mathrm{obese} \mathrm{middle-aged} \mathrm{adults} \mathrm{completed} \mathrm{a} \mathrm{1-year} \mathrm{weight} \mathrm{management}$ clinic. Participants met weekly for the first six months and bi-weekly thereafter. Meetings included instruction for diet, physical activity, and behavior change related to weight management. Additionally, participants followed a liquid very low-energy diet of $2092 \mathrm{~kJ}$ per day for the first three months of the study. Subsequently, participants followed a dietary plan for nine months that targeted a reduced percentage of carbohydrate ( 20\%) or fat ( 30\%) intake and an energy intake level calculated to maintain weight loss. Lipid subclasses using NMR spectroscopy were analyzed prior to weight loss and at multiple intervals during weight maintenance.

Results: Body weight change was not significantly different within or between groups during weight maintenance ( $p$ $>0.05$ ). The RC group showed significant increases in mean LDL size, large LDL, total HDL, large and small HDL, mean VLDL size, and large VLDL during weight maintenance while the RF group showed increases in total HDL, large and small HDL, total VLDL, and large, medium, and small VLDL $(p<0.05)$. Group*time interactions were significant for large and medium VLDL $(p>0.05)$.

Conclusion: Some individual lipid subclasses improved in both dietary groups. Large and medium VLDL subclasses increased to a greater extent across weight maintenance in the RF group.
\end{abstract}

\section{Introduction}

Low fat diets have been shown to reduce some risk factors associated with coronary heart disease (CHD) and have traditionally been considered the standard of care for dietary treatment in overweight and obese adults [1]. Recently, a low carbohydrate diet has emerged as a potentially viable alternative diet to reduce some risk factors for CHD. Compared to a traditional low fat diet, low carbohydrate diets are associated with greater weight loss over six months, decreased triglycerides, and increased HDL cholesterol while other important risk factors such as, total cholesterol and LDL cholesterol tend to be reduced

* Correspondence: lecheminant@byu.edu

1 Brigham Young University, 269 SFH, Provo, UT 84606, USA Full list of author information is available at the end of the article in similar fashion or reduction may be slightly greater on a low fat diet [2-7].

Subclasses for LDL cholesterol, HDL cholesterol, and VLDL cholesterol may provide a more complete cardiovascular risk profile than traditionally determined lipid values [8]. Recent investigations have reported subtle shifts in lipoprotein subclasses that tend to differ according to dietary carbohydrate and fat consumption [9-14]. As a result, there has been increased interest in how lipoprotein subclasses for LDL, HDL, and VLDL respond to manipulation of dietary carbohydrate and fat, particularly as it relates to CHD risk.

However, data comparing trends in lipoprotein subclasses according to carbohydrate and fat consumption are limited. In addition, existing studies comparing low 
carbohydrate and low fat diets have primarily reported lipoprotein subclasses during a period of weight loss and not weight stability [7]. The present study sought to improve upon these weaknesses in the literature. Therefore, the purpose of this study was to compare LDL, HDL, and VLDL subclasses in overweight/obese participants consuming either a reduced carbohydrate or reduced fat weight loss maintenance diet for nine months subsequent to three months of weight loss on a very low-energy diet (VLED). As there is currently no-agreed upon definition for a low carbohydrate diet [7] and the present study is not a very-low carbohydrate or ketogenic diet as reported elsewhere, the low carbohydrate and low fat diet groups will be described hereafter as reduced carbohydrate (RC) and reduced fat (RF) diet groups, respectively.

\section{Participants and Methods Participants}

This study received approval from the University Human Subjects Committee and all participants signed an approved informed consent before beginning. Participation criteria and methodology for this study have been reported elsewhere [15]. In addition, participants in the present study were taken from a larger study, but only participants who had complete lipid subclass measurements were used in this analysis. Participants were overweight or obese (BMI $>27 \mathrm{~kg} / \mathrm{m}^{2}$ ) but otherwise healthy and free of disease, middle-aged adults, and previously sedentary. Participants were excluded if they smoked, were participating in special diets, unable to perform moderate-intensity physical activity, pregnant or lactating, or in active psychological or psychiatric counseling.

\section{Study Design}

This study utilized a quasi-experimental design in which all participants were part of a behavioral weight management clinic and were prescribed a very low-energy diet (VLED) for three months followed by nine months in which participants consumed either a weight maintenance diet low in carbohydrate or low in fat. The rationale for this study design has been previously published [15].

\section{Intervention}

\section{Weight Management Clinics}

Participants for this study were part of a larger clinical weight management program that included a dietary, physical activity, and behavioral component. The protocol and description for these clinical programs have been previously published $[15,16]$. In short, participants met weekly in a small group format (15-20 individuals) for the first six months and bi-weekly during the final six months. The meeting protocol included: initial weigh-in for body weight, discussion of self-reported data for diet (liquid shakes, carbohydrate grams, or fat grams) and physical activity (minutes and pedometer counts), and instruction for diet, physical activity, or behavioral change strategies associated with weight management. As participants met separately by dietary group assignment, participants received diet-specific information and training during the weight maintenance period; otherwise, clinic meetings were identical between dietary groups.

\section{Very Low-Energy Diet}

During weight loss (months 1-3), a liquid VLED was utilized. Liquid supplements (Health Management Resources, Boston, MA) were taken at five different intervals daily totaling $\sim 2092 \mathrm{~kJ} /$ day. A vitamin and mineral supplement was taken twice per day along with the liquid supplements. As this was a weight-loss maintenance study, a minimum weight loss of $10 \%$ of initial body weight was required during VLED to progress to the weight maintenance stage (months 4-12). Participants reported their weekly liquid supplement total at each group meeting. In addition, the VLED period was medically managed by the study physician during its entire duration.

\section{Weight Maintenance Diets}

In order to decrease the likelihood of adverse events, participants progressively transitioned from liquid to solid foods, that were diet-specific (reduced carbohydrate or reduced fat), during the fourth month. By the end of month four, participants were solely consuming solid foods according to dietary group assignment and continued in this manner for the duration of weight maintenance. The weight maintenance energy level for each participant was determined utilizing the Harris-Benedict equation for resting metabolic rate and adjusted for physical activity using a factor of 1.4 [17]. In conjunction with the weight maintenance energy intake level, the RC diet participants were assigned $\sim 20 \%$ of their energy to be consumed from dietary carbohydrate and for the RF diet participants were assigned $\sim 30 \%$ of their energy to be consumed from dietary fat. To facilitate dietary tracking, each participant received an upper-limit daily gram level for either carbohydrate or fat consumption and recorded their gram consumption each day. Each participant reported their weekly carbohydrate or fat gram intake at the group meeting. In addition, gram intake was reported via email, phone, or fax if a meeting was missed and during the off-week when meetings were bi-weekly.

\section{Assessments}

\section{Anthropometrics}

At baseline and prior to each group meeting, body weight was assessed using a digital scale (Befour, Inc., Saukville, WI) accurate to $\pm 0.1 \mathrm{~kg}$. Participants were weighed in street clothes and without wearing shoes. Height was 
measured using a stadiometer (Perspective Enterprises, Portage, MI). Waist circumference was assessed at the narrowest portion of the waist between the lowest rib and iliac crest using a spring-loaded tape measure (Creative Health Products, Ann Arbor, MI) at baseline and throughout maintenance.

\section{Energy Intake}

Energy, carbohydrate, fat, and protein intake were assessed via 3-day food records at several intervals during weight maintenance. To complete 3-day food records, each participant recorded all food and beverages consumed during two weekdays and one weekend day. Participants were instructed to include as much detail as possible about all food and beverage items. A trained staff member reviewed and entered each dietary record and data were analyzed using the Nutrition Data System for Research (version 4.05_33).

\section{Blood Collection}

Overnight $12 \mathrm{hr}$ fasting blood samples were collected at baseline, and after three, six, and twelve months. All blood samples were collected into $10 \mathrm{ml}$ tubes containing EDTA using standard venipuncture methods by a trained phlebotomist. All samples were immediately separated by centrifugation for $15 \mathrm{~min}$ at $2000 \mathrm{~g}$. The separated plasma was transferred to cryogenic vials and stored at $-70^{\circ} \mathrm{C}$ for later analysis.

\section{Lipid Subclasses}

Fasting plasma samples were analyzed by LipoScience (LipoScience Inc, Raleigh, NC) using nuclear magnetic resonance spectroscopy (NMR) as described previously $[18,19]$. In brief, NMR identifies different lipoprotein subclasses based upon the idea that each lipoprotein particle emits a NMR signal that has a distinct frequency and shape. The intensities of these signals are proportional to the lipid mass concentration of that lipoprotein particle.

\section{Statistical Analysis}

The level of significance was set at $p<0.05$ for all statistical tests. The statistical software package PC-SAS (version 9.1, SAS Institute, Inc., Cary, NC) was used for tests of statistical significance. Independent T-tests were used to determine differences between groups at the beginning of weight loss and beginning of weight maintenance.
Mixed models were utilized to determine group differences, within group changes across time, and/or group*time interactions for anthropometrics, energy intake from 3-day food records, and lipid subclasses. Analysis of lipid subclasses for a group"time interaction were adjusted for the individual subclass and body weight at the beginning of weight maintenance. For analysis of 3day food records, adjusted means are reported to account for missing records. The General Linear Model was utilized to determine within group changes in weekly selfreported carbohydrate and fat grams across weight maintenance. When there was a missing data point for carbohydrate or fat gram intake in a particular week, the missing data was imputed by taking the average carbohydrate or fat gram from the previous month.

\section{Results}

In the $\mathrm{RC}$ group, 21 individuals completed all testing and assessments (12 women and 9 men) and in the RF group 14 individuals completed all testing and assessments (11 women and 3 men). Participants were primarily Caucasian (94\%). There were no significant differences at baseline between the RC group and RF group for body weight, BMI, or age (Table 1).

Weight loss during the VLED was significant. Participants assigned to the RC diet lost $\sim 20 \%$ of their body weight and the participants in the RF group lost 19\% of their body weight. Change in weight during the VLED was not different between groups $(p>0.05)$. Number of shakes during the VLED for all participants was $34.8 \pm 2.8$ equaling approximately $2092 \mathrm{~kJ}$ per day.

During weight maintenance, the RC group non-significantly increased body weight by $\sim 2.8 \%$. Likewise, the RF group showed a non-significant change of $\sim 3.0 \%$ in body weight across weight maintenance; however, change in body weight across the study was not different between groups $(p>0.05)$. Similar trends were observed for BMI.

The RC group averaged approximately $25 \%$ of energy consumed as dietary carbohydrate while the RF group averaged approximately $28 \%$ of energy consumed as dietary fat during weight maintenance (Table 2). As expected, there was a significant difference between the $\mathrm{RC}$ and RF groups for carbohydrate and fat intake during

Table 1: Baseline characteristics by group.

\begin{tabular}{ccccc}
\hline & $\begin{array}{c}\text { Reduced } \\
\text { Carbohydrate }\end{array}$ & Reduced Fat & $\boldsymbol{P}$ \\
$\mathbf{N}$ & 21 & 14 & & \\
Weight (kg) & $110.3 \pm 18.8$ & $112.3 \pm 17.6$ & 0.10 & 0.75 \\
BMI (kg/m2) & $38.5 \pm 4.5$ & $40.3 \pm 4.5$ & 1.41 & 0.25 \\
Age (y) & $50.9 \pm 8.9$ & $45.6 \pm 9.5$ & 2.87 & 0.10 \\
\hline
\end{tabular}


Table 2: Macronutrient composition by group during weight maintenance.

\begin{tabular}{lccc}
\hline & 4-Months & 6-Months & 12-Months \\
\hline Reduced Carb (n= 21) & & & $6774 \pm 458^{*+}$ \\
Energy Intake (kJ) & $5415 \pm 411$ & $6524 \pm 411$ & $104 \pm 10^{*+}$ \\
Carbohdyrates (g) & $76 \pm 9$ & $101 \pm 9$ & $90 \pm 8^{*+}$ \\
Fat (g) & $70 \pm 7$ & $83 \pm 7$ & $95 \pm 9$ \\
Protein (g) & $90 \pm 8$ & $100 \pm 8$ & \\
& & & $6500 \pm 771$ \\
Reduced Fat (n=14) & $7048 \pm 620$ & & $207 \pm 23$ \\
Energy Intake (kJ) & $224 \pm 18$ & $231 \pm 18$ & $52 \pm 9$ \\
Carbohdyrates (g) & $52 \pm 7$ & $58 \pm 7$ & $76 \pm 9$ \\
Fat (g) & $87 \pm 7$ & $89 \pm 7$ & \\
Protein (g) & & & \\
\hline
\end{tabular}

Mean \pm SE. Means are adjusted means.

*Indicates a significant difference within the Reduced Carbohydrate group over time. Changes were not significant over time for the Reduced Fat group.

†Indicates a significant difference from the Reduced Fat group.

weight maintenance (Table 2). There also was a significant difference in energy intake $(\mathrm{kJ})$ between groups. Food records revealed that carbohydrate consumption increased in the RC group during weight maintenance and fat consumption did not statistically change in the RF group during weight maintenance. Self-reported carbohydrate grams for the RC group averaged $88.1 \pm 32.7$ throughout weight maintenance. However, there was a statistically significant increase $(\sim 36 \%)$ in the number of self-reported carbohydrate grams consumed from the beginning of weight maintenance to the conclusion of the study $(p<0.0001)$ (Figure 1A). Self-reported fat grams for the RF group averaged $44.3 \pm 20.3$ throughout weight maintenance and did not significantly increase across the duration of weight maintenance $(p=0.152)$ (Figure 1B).

During the weight maintenance period, the $\mathrm{RC}$ group showed a significant increase in mean LDL size, large LDL, total HDL, large HDL, small HDL particle concentration, mean VLDL size, and large VLDL (Table 3). On the other hand, the RF group showed a significant increase in total $\mathrm{HDL}$, large $\mathrm{HDL}$, small $\mathrm{HDL}$, total VLDL, large VLDL, medium VLDL, and small VLDL particle concentration during the weight maintenance period. Tests for a group"time interaction revealed a significant interaction for large VLDL and medium VLDL, $\mathrm{p}$ $=0.044$ and $\mathrm{p}=0.049$, respectively (Table 3 ).

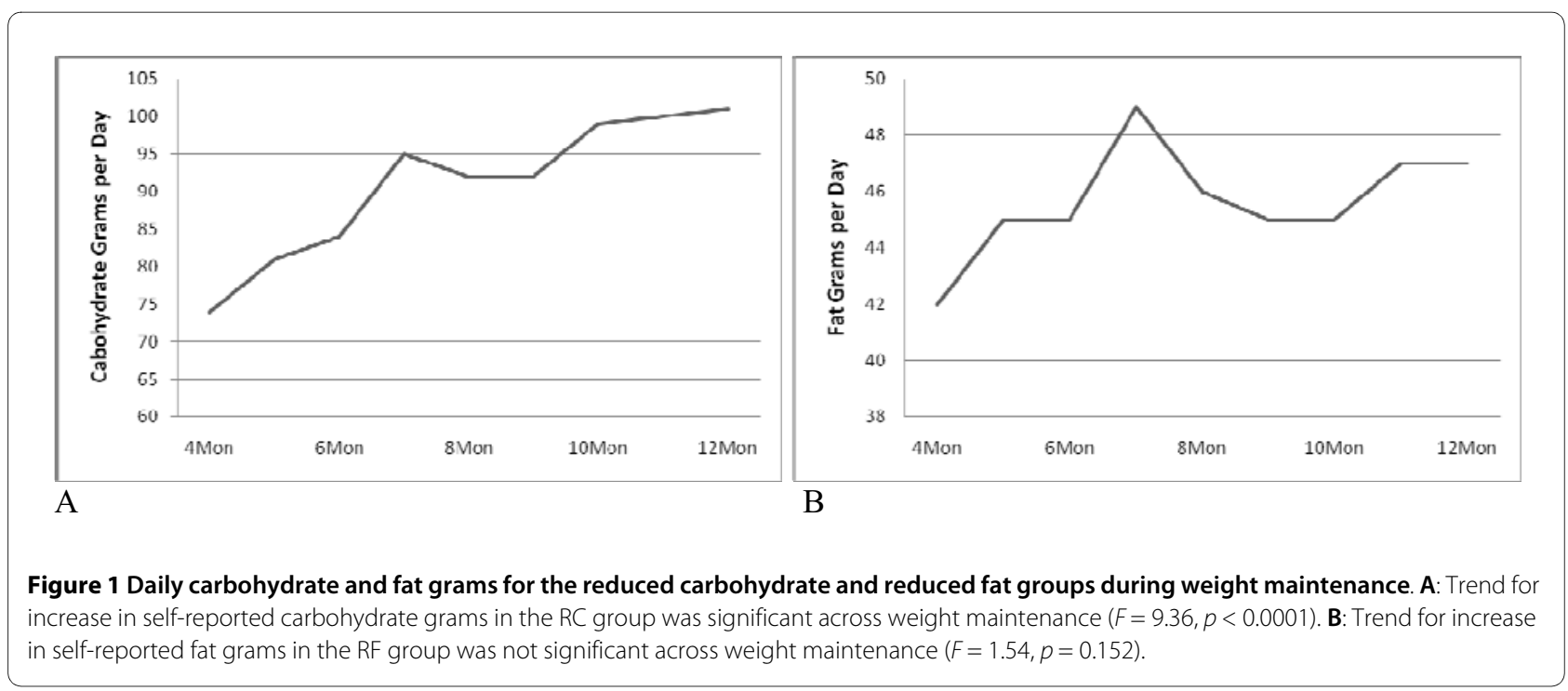


Table 3: Lipids subclasses across weight maintenance.

\begin{tabular}{|c|c|c|c|c|c|c|c|c|c|}
\hline \multirow[b]{3}{*}{ LDL } & \multicolumn{3}{|c|}{ Reduced Carbohydrate } & \multicolumn{6}{|c|}{ Reduced Fat } \\
\hline & 3-mon & 6-mon & 12-mon & $\begin{array}{l}\text { Difference } \\
12-3 \text { mon }\end{array}$ & 3-mon & 6-mon & 12-mon & $\begin{array}{l}\text { Difference } \\
12-3 \text { mon }\end{array}$ & $p+$ \\
\hline & & & & & & & & & \\
\hline $\begin{array}{l}\text { Mean LDL } \\
\text { Size }(\mathrm{nm})\end{array}$ & $20.9 \pm 0.7^{a}$ & $21.5 \pm 0.8^{b}$ & $21.2 \pm 0.7^{b}$ & $0.03^{*}$ & $20.9 \pm 0.4$ & $21.0 \pm 0.7$ & $21.0 \pm 0.8$ & 0.1 & 0.228 \\
\hline Total LDL & $1076 \pm 228$ & $1131 \pm 328$ & $1189 \pm 340$ & 113 & $1176 \pm 333$ & $1261 \pm 481$ & $1309 \pm 494$ & 133 & 0.945 \\
\hline Large LDL & $372 \pm 178^{a}$ & $544 \pm 260^{b}$ & $486 \pm 202^{b}$ & $114^{*}$ & $373 \pm 120$ & $423 \pm 139$ & $416 \pm 185$ & 43 & 0.131 \\
\hline Small LDL & $663 \pm 196$ & $547 \pm 382$ & $671 \pm 364$ & 8 & $737 \pm 260$ & $782 \pm 480$ & $814 \pm 564$ & 77 & 0.347 \\
\hline $\begin{array}{l}\text { Medium- } \\
\text { Small LDL }\end{array}$ & $133 \pm 37$ & $111 \pm 78$ & $139 \pm 79$ & 6 & $152 \pm 52$ & $162 \pm 99$ & $177 \pm 123$ & 25 & 0.407 \\
\hline $\begin{array}{l}\text { Very-Small } \\
\text { LDL }\end{array}$ & $530 \pm 163$ & $436 \pm 304$ & $532 \pm 287$ & 2 & $585 \pm 208$ & $621 \pm 382$ & $637 \pm 441$ & 52 & 0.334 \\
\hline \multicolumn{10}{|l|}{ HDL } \\
\hline $\begin{array}{l}\text { Mean HDL } \\
\text { Size }(\mathrm{nm})\end{array}$ & $9.2 \pm 0.4$ & $9.3 \pm 0.4$ & $9.15 \pm 0.4$ & -0.05 & $9.1 \pm 0.3$ & $9.0 \pm 0.4$ & $9.1 \pm 0.4$ & 0 & 0.282 \\
\hline Total HDL & $25.7 \pm 4.3^{a}$ & $33.0 \pm 5.9^{b}$ & $36.3 \pm 5.7 c$ & $10.6^{*}$ & $27.6 \pm 3.6^{a}$ & $34.7 \pm 6.0^{\mathrm{b}}$ & $37.4 \pm 4.6^{c}$ & $9.8^{*}$ & 0.845 \\
\hline Large HDL & $5.9 \pm 1.9^{a}$ & $8.2 \pm 3.0^{\mathrm{b}}$ & $8.8 \pm 3.3^{b}$ & $2.9^{*}$ & $6.1 \pm 2.1^{\mathrm{a}}$ & $7.6 \pm 3.4^{b}$ & $8.6 \pm 4.0^{\mathrm{b}}$ & $2.5^{*}$ & 0.530 \\
\hline $\begin{array}{l}\text { Medium } \\
\text { HDL }\end{array}$ & $3.6 \pm 2.6$ & $2.5 \pm 3.2$ & $2.7 \pm 2.8$ & -0.9 & $4.0 \pm 2.5$ & $4.9 \pm 3.8$ & $4.8 \pm 3.5$ & 0.8 & 0.112 \\
\hline $\begin{array}{c}\text { Small HDL } \\
\text { VLDL }\end{array}$ & $16.2 \pm 4.3^{a}$ & $22.3 \pm 4.9^{b}$ & $24.8 \pm 5.0^{c}$ & $8.6^{*}$ & $17.5 \pm 3.9^{a}$ & $22.2 \pm 6.5^{b}$ & $24.0 \pm 5.2^{b}$ & $6.5^{*}$ & 0.441 \\
\hline $\begin{array}{l}\text { Mean VLDL } \\
\text { Size }(n m)\end{array}$ & $43.1 \pm 3.5^{\mathrm{a}}$ & $44.6 \pm 5.1^{a}$ & $48.4 \pm 9.9^{b}$ & $5.3^{*}$ & $43.8 \pm 5.6$ & $47.3 \pm 7.4$ & $47.7 \pm 11.8$ & 3.9 & 0.445 \\
\hline Total VLDL & $44.3 \pm 26.5$ & $49.4 \pm 28.5$ & $50.5 \pm 29.8$ & 6.2 & $56.2 \pm 30.0^{a}$ & $77.1 \pm 32.2^{b}$ & $76.0 \pm 30.1^{b}$ & $19.8^{*}$ & 0.194 \\
\hline Large VLDL & $0.7 \pm 0.8^{a}$ & $0.6 \pm 0.8^{a}$ & $1.5 \pm 1.9^{b}$ & $0.8^{*}$ & $1.7 \pm 2.5^{\mathrm{a}}$ & $3.7 \pm 3.9^{b}$ & $3.5 \pm 4.9^{b}$ & $1.8^{*}$ & 0.044 \\
\hline $\begin{array}{l}\text { Medium } \\
\text { VLDL }\end{array}$ & $13.3 \pm 11.5$ & $11.7 \pm 10.0$ & $15.5 \pm 13.3$ & 2.2 & $17.5 \pm 13.5^{a}$ & $26.9 \pm 13.5^{b}$ & $25.6 \pm 16.5^{b}$ & $8.1^{*}$ & 0.049 \\
\hline Small VLDL & $30.3 \pm 16.8$ & $37.1 \pm 21.3$ & $33.5 \pm 18.8$ & 3.2 & $37.0 \pm 17.4^{a}$ & $46.5 \pm 17.4^{b}$ & $46.9 \pm 16.9^{b}$ & $9.9^{*}$ & 0.590 \\
\hline
\end{tabular}

\section{Discussion}

This study is unique as the RC and RF diets were consumed for weight maintenance rather than weight loss thereby reducing the effect of body weight change on the lipid outcomes measured. Taken as a whole, lipid subclass changes were similar between dietary groups across the duration of the study; however, a RC diet significantly attenuated the increase in some VLDL outcomes compared to the RF diet.

While the RF diet in the present study was consistent with the standard definition of a low fat diet $(<30 \%)$, carbohydrate consumption in the RC group tended to be higher than some other studies in the literature. Accordingly, previous low carbohydrate and low fat studies have found different results in lipid subclasses compared to the present study $[9,11,13]$. It is possible that a moderate reduction in carbohydrate intake, as observed in the present study, is insufficient to produce more significant changes in lipid subclasses compared to a standard low fat diet when body weight is controlled. However, in a study by Seshadri et al., a carbohydrate content of $\leq 30$ grams per day was prescribed for the low carbohydrate group but actual reported carbohydrate intake was $32 \pm$ $20 \%$ ( $113 \mathrm{~g}$ of carbohydrate per day) at the 6-month fol- 
low-up period [10]. It is noteworthy that Seshadri et al. reported somewhat similar lipid subclass trends to the present study, for example, a significant difference in change in large VLDL and lack of a significant difference in change in most other lipid subclasses between the low carbohydrate and low fat groups [10]. Overall, this begs the question of whether or not a carbohydrate consumption threshold exists to produce lipid subclass changes, beyond VLDL, that differ from those of a low fat diet.

Further, it is noteworthy that in the present study dietary carbohydrate consumption significantly increased across weight maintenance in the RC group ( 36\%) while dietary fat consumption did not statistically change in the RF group. Given the gradual and significant increase in carbohydrate content over time in the RC group, it is possible that lipid subclass changes would have been different had the RC group not increased their daily carbohydrate consumption; however, this is somewhat speculative.

While this study has the strength of being one of the first to compare a RC and RF diet for an extended period of weight maintenance (9 months) there also are limitations. Specifically, sample size was small. As a result, these data from may be most appropriately considered as pilot data for future studies. In addition, this study was quasi-experimental. This study design introduces the possibility that other factors or biases existed and influenced the results of the study.

In summary, this study suggests that following three months of significant weight loss on a VLED, a weight maintenance RC and RF diet show similar changes for lipid subclasses; however, medium VLDL and large VLDL did not increase to the same extent on the RC diet as the $\mathrm{RF}$ diet. A larger sample and stricter adherence to the RC diet may have altered the results of the study and may have yielded results that better differentiate between these diets for lipid subclass outcomes and potentially for CHD risk; this deserves further investigation.

\section{List of Abbreviations}

RC: Reduced carbohydrate; RF: Reduced fat; LDL: Lowdensity lipoprotein; HDL: High-density lipoprotein; VLDL: Very low-density lipoprotein; CHD: Coronary heart disease; VLED: Very low-energy diet; NMR: Nuclear magnetic resonance spectroscopy.

\section{Competing interests}

Dr. Mary C. Vernon received honoraria as a consultant for Mrs. Veronica Atkins, Chairperson of the Board of Directors for the Robert C. Atkins Foundation.

\section{Authors' contributions}

$J D$ conceived the study design with input from JL, BS, MV, and EW. JL coordinated and conducted the overall study with input from BS, EW, MV, and JD. MV provided medical oversight of all subjects. Data were analyzed by $J$ and BS. The manuscript was prepared by $J \mathrm{~L}, \mathrm{BS}, \mathrm{JD}$, and all authors contributed to the editing and input of the final manuscript.

\section{Acknowledgements}

This study was partially supported by Health Management Resources and the Robert C. Atkins Foundation.

\section{Author Details}

1Brigham Young University, 269 SFH, Provo, UT 84606, USA, 2The University of Kansas, 1301 Sunnyside Avenue, Lawrence, Kansas 66045, USA, ${ }^{3}$ Department of Medicine, Duke University Medical Center, 4020 N Roxboro Street, Durham, North Carolina 27704, USA, 4Private Practice, 21624 Midland Drive, Shawnee, Kansas 66218, Lawrence, Kansas, USA and ${ }^{5}$ Center for Physical Activity and Weight Management, The University of Kansas, 1301 Sunnyside Avenue, Lawrence, Kansas 66045, USA

Received: 26 March 2010 Accepted: 1 June 2010

Published: 1 June 2010

\section{References}

1. Heart National, Lung and Blood Institute: Clinical guidelines on the identification, evaluation, and treatment of overweight and obesity in adults: executive summary. Expert Panel on the Identification, Evaluation, and Treatment of Overweight in Adults. Am J Clin Nutr 1998, 68:899-917.

2. Brehm BJ, Seeley RJ, Daniels SR, D'Alessio DA: A randomized trial comparing a very low carbohydrate diet and a calorie-restricted low fat diet on body weight and cardiovascular risk factors in healthy women. J Clin Endocrinol Metab 2003, 88:1617-1623.

3. Foster GD, Wyatt HR, Hill JO, McGuckin BG, Brill C, Mohammed BS, Szapary PO, Rader DJ, Edman JS, Klein S: A randomized trial of a lowcarbohydrate diet for obesity. N Eng/ J Med 2003, 348:2082-2090.

4. Stern L, labal N, Seshadri P, Chicano KL, Daily DA, McGrory J, Williams M, Gracely EJ, Samaha FF: The effects of low-carbohydrate versus conventional weight loss diets in severely obese adults: one-year follow-up of a randomized trial. Ann Intern Med 2004, 140:778-785.

5. Yancy WS Jr, Olsen MK, Guyton JR, Bakst RP, Westman EC: A lowcarbohydrate, ketogenic diet versus a low-fat diet to treat obesity and hyperlipidemia: a randomized, controlled trial. Ann Intern Med 2004, 140:769-777.

6. Gardner CD, Kiazand A, Alhassan S, Kim S, Stafford RS, Balise RR, Kraemer $\mathrm{HC}$, King AC: Comparison of the Atkins, Zone, Ornish, and LEARN diets for change in weight and related risk factors among overweight premenopausal women: the A TO Z Weight Loss Study: a randomized trial. Jama 2007, 297:969-977

7. Samaha FF, Foster GD, Makris AP: Low-carbohydrate diets, obesity, and metabolic risk factors for cardiovascular disease. Curr Atheroscler Rep 2007, 9:441-447

8. Koba S, Yokota Y, Hirano T, Ito Y, Ban Y, Tsunoda F, Sato T, Shoji M, Suzuki $\mathrm{H}$, Geshi E, et al: Small LDL-cholesterol is superior to LDL-cholesterol for determining severe coronary atherosclerosis. J Atheroscler Thromb 2008, 15:250-260.

9. Sharman MJ, Kraemer WJ, Love DM, Avery NG, Gomez AL, Scheett TP, Volek JS: A ketogenic diet favorably affects serum biomarkers for cardiovascular disease in normal-weight men. J Nutr 2002, 132:1879-1885.

10. Seshadri P, Iqbal N, Stern L, Williams M, Chicano KL, Daily DA, McGrory J, Gracely EJ, Rader DJ, Samaha FF: A randomized study comparing the effects of a low-carbohydrate diet and a conventional diet on lipoprotein subfractions and C-reactive protein levels in patients with severe obesity. Am J Med 2004, 117:398-405.

11. Wood RJ, Volek JS, Liu Y, Shachter NS, Contois JH, Fernandez ML: Carbohydrate restriction alters lipoprotein metabolism by modifying VLDL, LDL, and HDL subfraction distribution and size in overweight men. J Nutr 2006, 136:384-389.

12. Krauss RM, Blanche PJ, Rawlings RS, Fernstrom HS, Williams PT: Separate effects of reduced carbohydrate intake and weight loss on atherogenic dyslipidemia. Am J Clin Nutr 2006, 83:1025-1031. quiz 1205

13. Volek JS, Sharman MJ, Gomez AL, DiPasquale C, Roti M, Pumerantz A, Kraemer WJ: Comparison of a very low-carbohydrate and low-fat diet on fasting lipids, LDL subclasses, insulin resistance, and postprandial lipemic responses in overweight women. J Am Coll Nutr 2004, 23:177-184. 
14. Westman EC, Yancy WS Jr, Olsen MK, Dudley T, Guyton JR: Effect of a lowcarbohydrate, ketogenic diet program compared to a low-fat diet on fasting lipoprotein subclasses. Int J Cardiol 2006, 110:212-216.

15. LeCheminant JD, Gibson CA, Sullivan DK, Hall S, Washburn R, Vernon MC, Curry C, Stewart E, Westman EC, Donnelly JE: Comparison of a low carbohydrate and low fat diet for weight maintenance in overweight or obese adults enrolled in a clinical weight management program. Nutr J 2007, 6:36.

16. LeCheminant JD, Jacobsen DJ, Hall MA, Donnelly JE: A comparison of meal replacements and medication in weight maintenance after weight loss. J Am Coll Nutr 2005, 24:347-353.

17. Lin PH, Proschan MA, Bray GA, Fernandez CP, Hoben K, Most-Windhauser $M$, Karanja N, Obarzanek E: Estimation of energy requirements in a controlled feeding trial. Am J Clin Nutr 2003, 77:639-645.

18. Otvos JD: Measurement of lipoprotein subclass profiles by nuclear magnetic resonance spectroscopy. In Handbook of LipoProtein Testing 2nd edition. Washington DC: AACC Press; 2000.

19. Otvos JD, Jeyarajah EJ, Bennett DW, Krauss RM: Development of a proton nuclear magnetic resonance spectroscopic method for determining plasma lipoprotein concentrations and subspecies distributions from a single, rapid measurement. Clin Chem 1992, 38:1632-1638.

doi: $10.1186 / 1476-511 X-9-54$

Cite this article as: LeCheminant et al., Comparison of a reduced carbohydrate and reduced fat diet for $L D L, H D L$, and VLDL subclasses during 9months of weight maintenance subsequent to weight loss Lipids in Health and Disease 2010, 9:54

Submit your next manuscript to BioMed Central and take full advantage of:

- Convenient online submission

- Thorough peer review

- No space constraints or color figure charges

- Immediate publication on acceptance

- Inclusion in PubMed, CAS, Scopus and Google Scholar

- Research which is freely available for redistribution

Submit your manuscript at www.biomedcentral.com/submit
Ciomed Central 